\title{
Research on Company Operation Performance Evaluation Based on BP Neural Network
}

\author{
Lili Zhang, Weiping Zhong \\ Department of Economics, Qinhuangdao Institute of Technology, Qinhuangdao, 066000, China
}

Keywords: Operation performance, BP neural network, Data envelopment analysis, Comprehensive evaluation

\begin{abstract}
For operation performance evaluation of company mainly uses linear or nonlinear evaluation model. The nonlinear model can better simulate the economic phenomena, and its evaluation results are more objective, accurate and have more practical reference value. From the assessment of the input-output efficiency perspective, this paper build the evaluation index system based on basic financial indicators. On this basis, the high-tech company operation performance evaluation model is established based on BP neural network. 20 listed companies' financial data in 2012 are regarded as the training and testing samples of neural network. The trained BP neural network are applied in evaluating the company operation performance, and its empirical results are satisfactory.
\end{abstract}

\section{Introduction}

The long-term operation performance depend the size of the enterprise value. Operation performance evaluation means objectively, fairly and accurate evaluating the company operation performance through using mathematical statistics and operations research principles. In this process, a specific index system will be established. Moreover, the control uniform standards and the quantitative and qualitative analysis will also applied in the model. The listed company's operation performance evaluation should be considered from many aspects and indicators, for a single financial indicator can only reflect the company's business activities at a moment or some aspects of the case. Evaluating operation performance is a complex project which involves all aspects of business[1,2].

\section{Establishment of company operation performance evaluation index system}

In the process of evaluating the company operation performance, non-dimensional treatment should be regarded as the basis of the system evaluation model. In addition, qualitative analysis and quantitative analysis should also be applied in collecting and analyzing the scale, level, structure and efficiency of a large number of companies. The system architecture, subsystems and system functions collaborative evolution of environmental effects in the system is necessary for us to understand, which fully reflects specific status and role in a certain condition. Corporate investors and operators need to comprehensively evaluate the financial situation of their enterprises, and the evaluation results can be as a basis for investment and business decisions. Based on this, the company operation performance evaluation index system is established from the perspective of the input-output assessment of business performance, which is shown in Table 1 [3].

\section{BP neural network}

Neural network is essentially a simulation system for the human brain's thinking process. Its core is the mathematical models and algorithms, and the physical implementation is computer software. Just like thins are composed of atoms, neural network is composed of many interconnected neurons[4-6]. Fig. 1 shows the basic work principle of neurons, and the mathematical expression is as follows:

$$
y=f\left(\sum w_{i} x_{i}\right)
$$


Table 1. Company operation performance evaluation index system

\begin{tabular}{cc}
\hline Index type & Index name \\
\hline & Net assets per share in early B1 \\
Tnput indicators A1 & Main business costs B3 \\
& The total cost of the annual salary \\
B4 \\
Net assets per share at the end B5 \\
Total asset value per share at the \\
end B6 \\
Output indicators A2 \\
Main business income B7 \\
Net profit after tax B8 \\
Return on total assets B9 \\
Return on equity B10 \\
\hline
\end{tabular}

Where $x_{i}$ and $y$ are respectively the input and output. $w_{i}$ is weight coefficient. $f$ is characteristic function, which reflects the mapping relationship between inputs and outputs, and it is usually a nonlinear function.

This seemingly simple model will produce a powerful neural network combing different network topology and network algorithms together. Neural network consists of input layer, hidden layer and output layer, which is shown in Fig. 1.

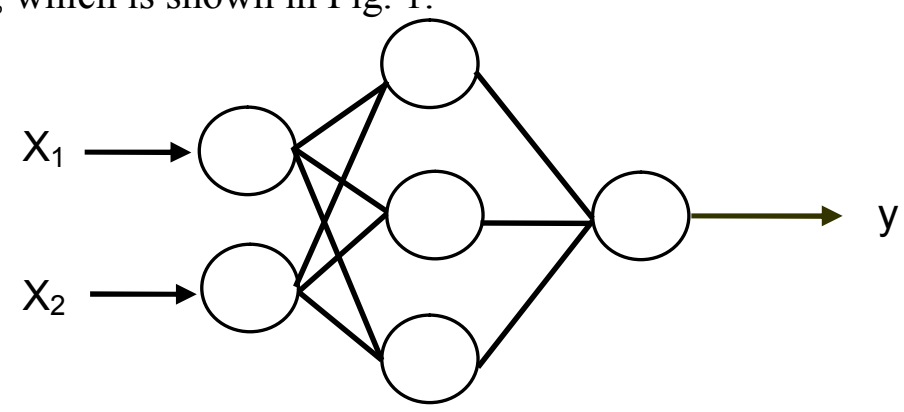

Fig. 1 The connection model of neural network

With a lot of development of neural networks, it was found that no matter how the organizational structure of the network it is, It is always has the following two characteristics:

(1) Self-learning

Neural networks can be modified according to the external environment of their own behavior in order to adapt to the external environment, which is mainly due to its learning process. Learning is often the first step in using neural networks. When a group of information is input, neural networks can continue to adjust its internal parameters (or say weighting coefficient), and eventually produce a series of consistent output.

(2) Generation

Once after the self-learning, the response of neural network, to some extent, to the reducing of input information and their own local defects are no longer sensitive. This mechanism can make the neural network has a strong fault tolerance and reduce the input data quality requirements.

The increasing function means that the multiplications of a node's all input and its corresponding connection are all added up. For example, the increasing function value of the first node in hidden layer is defined as below.

$$
x_{1}=\sum_{i} D_{i} w_{i 1}
$$

Where $x_{1}$ is the input value of the first node in hidden layer. $D_{i}$ is the input data of the $i$ th node in input layer. $w_{i 1}$ is the weight value from the $i$ th node in input layer to the first node in hidden layer. 
The activation function means that the added value mentioned above function are transformed to the output value of the node through a certain mathematical method. For example, the output value of the first node in hidden layer is defined as below.

$$
y_{1}=g\left(x_{1}\right)
$$

Where $g(x)$ is named as activation function. The common activation functions include Sigmoid function, proportional function, saturation function, hyperbolic functions and so on.

\section{Operation performance evaluation model based on BP neural network}

For operation performance evaluation relative efficiency, many scholars have proposed using data envelopment analysis (DEA) method to carry [7,8]. DEA method has its own advantages in dealing with multi- input and multi -output problem. It can avoid the influence of subjective factors in large part to and its evaluation results are more scientific and accurate. However, DEA also has its own shortcomings. Compared with DEA, BP neural network has the absolute advantage over than DEA in handling complex, multi-dimensional nonlinear problems, and better able to make up for deficiencies DEA. Thus, three layers BP neural network is used in this paper to evaluate company operation performance.

Role function reflects neural network input node in the upper lower the strength of the stimulus pulse function, also known as stimulation function. This paper select Sigmoid function which contains the continuous values from 0 to 1 .

$$
f(x)=\frac{1}{1+e^{-x}} \text {. }
$$

Determining the number of hidden layer units and the number of input and output units are directly related. If hidden layer unit is too small, the network will not be trained, or the network will not be strong, which can not identify the samples not seen before. If hidden layer unit makes learning much too long, the error may not be significantly reduced. Generally, the number of hidden layer unit can can be calculated according to Eq.(5).

$$
n_{H}=\sqrt{n_{l}+n_{o}}+l \text {. }
$$

Where $n_{H}$ represents the number of hidden layer unit, $n_{l}$ represents the number of input layer unit, $n_{o}$ represents the number of output layer unit, $l$ represents a random integer from 1 to 10 .

\section{Specific example and result analysis}

20 listed companies are selected in this paper as analysis units, and BP neural network method is applied in evaluating the company operation performance [9].

(1) Dimensionless processing for evaluation index values

Eliminate the dimensions of evaluation indexes according to Eq.(6). The actual index value

$x_{i j}$ should be converted into evaluation value which are from 0.1 to 1 . The range and standardized calculation formula are respectively shown as Eq.(6) and Eq.(7).

$$
\begin{aligned}
& d_{i}=\frac{\max \left(x_{i j}\right)-\min \left(x_{i j}\right)}{a_{\max }-a_{\min }} . \\
& x_{i j}=\frac{x_{i j}-\min \left(x_{i j}\right)}{d_{j}}+0.1
\end{aligned}
$$

Where $a_{\max }$ equals 1 and $a_{\min }$ equals 0.1 .

(2) Neural network training mode 
10 input and output indicators of listed companies shown in Table 1 are regarded as the training unit input data, while the corresponding evaluation value of the company operation performance are regarded as the output data. The original evaluation indicators are classified by theirs correlation which shown in Table 2. Then the normalized data completes the information synthesis of index through principal component analysis. The synthetic indicators data are input in mixed DEA model. The evaluation values of company operation performance can be calculated based on the mixed DEA.

Table 2. Evaluation correlation classification

\begin{tabular}{ccc}
\hline Index type & Relativity type & Index label \\
\hline Input indicators & $\begin{array}{c}\text { Relative amounts } \\
\text { part } \\
\text { Absolute amounts } \\
\text { part } \\
\text { Relative amounts } \\
\text { part 1 } \\
\text { Output indicators }\end{array}$ & Belative amounts \\
part 2 & B3, B4 \\
& $\begin{array}{c}\text { Absolute amounts } \\
\text { part }\end{array}$ & B5, B6 \\
\end{tabular}

(3) Network training and testing

10 standardization index data of listed companies and mixed DEA model to calculate the value of the evaluation network composed of 20 input and output data model. The number 1 to 16 of the companies are selected as the training sample of the BP neural network. If the hidden layer of a forward neural network has a proper number of units, this proposed network can be used to very accurately simulate any function, which a lot about cutting-edge research in neural networks can be reflected. The number of neural network hidden layer units can be determined according to Eq.(2). In this paper, the hidden layer unit is determined as 16. Thus, the network structure of BP neural network are defined as 10-16-1. The learning accuracy $E$ is defined as 0.000001 , and learning speed $I r$ is defined as 0.05 . Performance evaluation value of each training unit is regarded as the desired output. The number 17 to 20 of the companies are selected as the testing sample of the BP neural network. The testing results are shown in Table 3.

Table 3. BP neural network testing results

\begin{tabular}{ccccc}
\hline $\begin{array}{c}\text { Company } \\
\text { label }\end{array}$ & $\begin{array}{c}\text { Desired } \\
\text { output }\end{array}$ & $\begin{array}{c}\text { Forecast } \\
\text { data }\end{array}$ & $\begin{array}{c}\text { Test data } \\
\text { errors }\end{array}$ & $\begin{array}{c}\text { Percentage } \\
\text { deviation } \\
\text { data }\end{array}$ \\
\hline 17 & 0.667 & 1 & 1 & 0.612 \\
18 & 0.664 & 0.956 & 0.999 & 0.698 \\
19 & 0.003 & 0.014 & 0.001 & 0.006 \\
20 & 0.507 & 1.432 & 0.14 & 1.039 \\
\hline
\end{tabular}

As is seen in Table 3, the testing results of BP neural network are very close to the desired output. The largest relative error is only 1.039 , and the lowest relative error is 0.612 , which can conclude that the proposed model has a high accuracy in evaluating the company operation performance.

\section{Conclusion}

The company operation performance evaluation model based on BP neural network is established in the paper. 20 listed companies are selected as the sample of BP neural network for analysis. Through training the network, the proposed network has a high forecasting accuracy, and its evaluation results are objective and accurate. In practice, determine the evaluation criteria, select industry outstanding value, the similar enterprises can carry out analysis, grasp the operating status 
of enterprises in the industry, which provides an important reference for decision-making for companies.

\section{References}

[1] Shiuwan Hung, Dongsing He, Wenmin Lu. Evaluating the dynamic performance of business groups from the carry-over perspective: A case study of Taiwan's semi conductor industry. Omega. 46, pp.1-10, 2014.

[2] Xuefeng Zhao, Qin Ba, Lei Zhou, Weijie Li, Jinping Ou. BP neural network recognition algorithm for scour monitoring of subsea pipelines based on active thermometry. International Journal for Light and Electron Optics. 125(18), pp. 5426-5431, 2014.

[3] Esmaiel Keshavarz, Mehdi Toloo. Finding efficient assignments: An innovative DEA approach. Measurement. 58, pp. 448-458, 2014.

[4] Hu Junguo, Zhou Guomo, Xu Xiaojun. Using an improved back propagation neural network to study spatial distribution of sunshine illumination from sensor network data. Ecological Modelling. 266(24), pp. 86-96, 2013.

[5] Jaehun Park, Dongha Lee, Joe Zhu. An integrated approach for ship block manufacturing process performance evaluation: Case from a Korean shipbuilding company. International Journal of Production Economics. 156, pp. 214-222, 2014.

[6] João Malta, Pedro F. Cunha. A new approach for cost modelling and performance evaluation within operations planning. CIRP Journal of Manufacturing Science and Technology. 4, pp. 234-242, 2011.

[7] Jia Zhengyuan, Wang Chunmei, Huang Zhiwei, Zhang Gang. Evaluation research of regional power grid companies' operation capacity based on entropy weight fuzzy comprehensive model. Procedia Engineering. 15, pp. 4626-4630, 2011.

[8] Shiwei Yu, Kejun Zhu, Fengqin Diao. A dynamic all parameters adaptive BP neural networks model and its application on oil reservoir prediction. Applied Mathematics and Computation. 195, pp. 66-75, 2008.

[9] Tuo zhong, Wang li-yuan. Improved BP Neural Network's Application in the Bank Early Warning. Procedia Engineering. 23, pp. 216-221, 2011. 